\title{
Diagnostic performance of ATA, BTA and TIRADS sonographic patterns in the prediction of malignancy in histologically proven thyroid nodules
}

\author{
Chiaw Ling $\underline{\text { Chng }}{ }^{1}$, MBBS, MRCP, Hong Chang $\underline{T a n}^{1}$, MBBS, MRCP, Chow Wei $\underline{T o O}^{2}$, MBBS, FRCR, Wei Ying $\underline{\operatorname{Lim}}^{1}$, MBBS, MRCP, \\ Priscilla Pei Sze Chiam ${ }^{1}$, MBChB, MRCP, Ling $\underline{\text { hhu }}^{1}$, MBBChir, MRCP, Nivedita Vikas Nadkarni ${ }^{3}$, PhD, Adoree Yi Ying $\underline{\text { Lim }}^{1}$, MBBS, MRCP
}

\begin{abstract}
INTRODUCTION We aimed to compare the malignancy risk stratification of histologically proven thyroid nodules using the 2015 American Thyroid Association (ATA) Management Guidelines, 2014 British Thyroid Association (BTA) Guidelines for the Management of Thyroid Cancer and the Thyroid Imaging Reporting and Data System (TIRADS).

METHODS Thyroid nodules measuring $>1 \mathrm{~cm}$ resected over 5.5 years were retrospectively studied. Demographic information as well as cytology and histopathology results were collected. Static ultrasonography (US) images and radiologists' reports of each resected nodules were reviewed and classified based on the above risk classification systems. RESULTS A total of 167 thyroid nodules from 150 patients were examined. More malignant nodules were solid (78.4\% vs. $62.5 \% ; p=0.049)$ or hypoechoic $(70.6 \%$ vs. $28.6 \% ; p<0.001)$, and had irregular margins $(35.3 \%$ vs. $8.0 \% ; p<0.001)$, taller-than-wide morphology ( $9.8 \%$ vs. $2.7 \%$; $p=0.031)$, microcalcifications $(33.3 \%$ vs. $8.0 \% ; p<0.001)$, disrupted rim calcifications ( $9.8 \%$ vs. $0.9 \% ; p=0.012)$ or associated abnormal cervical lymphadenopathy $(13.7 \%$ vs. $0.9 \% ; p=0.001)$ compared with benign nodules. The guidelines' diagnostic performance was: ATA - sensitivity $98.0 \%$, specificity $17.3 \%$, positive predictive value (PPV) 35.0\%, negative predictive value (NPV) $95.0 \%$; BTA - sensitivity $90 \%$, specificity $50.9 \%$, PPV 45.5\%, NPV 91.8\%; and TIRADS - sensitivity $94.0 \%$, specificity $28.2 \%$, PPV $37.3 \% \%$, NPV $91.2 \%$.

CONCLUSION Sonographic patterns outlined by the three guidelines displayed high sensitivity and NPV. Although isolated suspicious US features cannot predict malignancy risk, they should be considered when risk stratifying nodules that do not fit into particular sonographic patterns based on current guidelines.
\end{abstract}

Keywords: thyroid cancer, thyroid nodule, ultrasonography

\section{INTRODUCTION}

Fine needle aspiration (FNA) is a standard initial step and an invaluable tool in the assessment and evaluation of thyroid nodules. The addition of ultrasonography guidance in FNA (USFNA) has been shown to improve the accuracy of the procedure and decrease the false-negative rate of a benign cytology diagnosis. ${ }^{(1,2)}$ While it has been reported that certain sonographic features of a thyroid nodule are associated with an increased likelihood of malignancy, no single predictor has been found to have a high positive predictive value (PPV) for cancer. Hence, many professional bodies have striven to produce guidelines to aid clinical decisions in the selection of thyroid nodules for US-FNA, recommending that only nodules with suspicious US characteristics be biopsied.

In recent years, both the American Thyroid Association (ATA) and British Thyroid Association (BTA) have published guidelines for US risk stratification of thyroid nodules. ${ }^{(3,4)}$ Importantly, both guidelines emphasised sonographic patterns, rather than growth of thyroid nodules, as the major deciding factor for biopsy. The Thyroid Imaging Reporting and Data System (TIRADS), first proposed by Horvath et al, (5) was introduced to improve communication between radiologists and physicians through a standardised reporting format. Since its introduction, many variations of TIRADS have been proposed. The TIRADS structure suggested by Kwak et $\mathrm{al}^{(6)}$ predicts malignancy risks according to only the number of suspicious US features, and several studies have proven that these US features are effective not only in risk stratification, but also in patient treatment. ${ }^{(7-9)}$ However, it is often difficult to compare the accuracy of different guidelines due to the differing samples of the various studies from which the guidelines were established.

The aim of the present study was to compare the malignancy risk stratification of histologically proven thyroid nodules using the 2015 ATA Management Guidelines for Adult Patients with Thyroid Nodules and Differentiated Thyroid Cancer, ${ }^{(3)}$ the 2014 BTA Guidelines for the Management of Thyroid Cancer ${ }^{(4)}$ and the TIRADS classification proposed by Kwak et al. ${ }^{(6)}$ We applied these three sets of US risk classification systems to the same sample to assess their diagnostic performance in the prediction of histologically confirmed malignancy.

\section{METHODS}

A total of 1,531 thyroid nodules underwent US-FNA at the Department of Endocrinology, Singapore General Hospital, Singapore, between January 2010 and June 2015. The decision to perform a biopsy on these nodules during the study period was

${ }^{1}$ Department of Endocrinology, ${ }^{2}$ Department of Vascular and Interventional Radiology, Singapore General Hospital, ${ }^{3}$ Centre for Quantitative Medicine, Duke-NUS Medical School, Singapore

Correspondence: Dr Chng Chiaw Ling, Senior Consultant, Department of Endocrinology, Singapore General Hospital, Level 3, Academia, 20 College Road, Singapore 169856. chng.chiaw.ling@singhealth.com.sg 

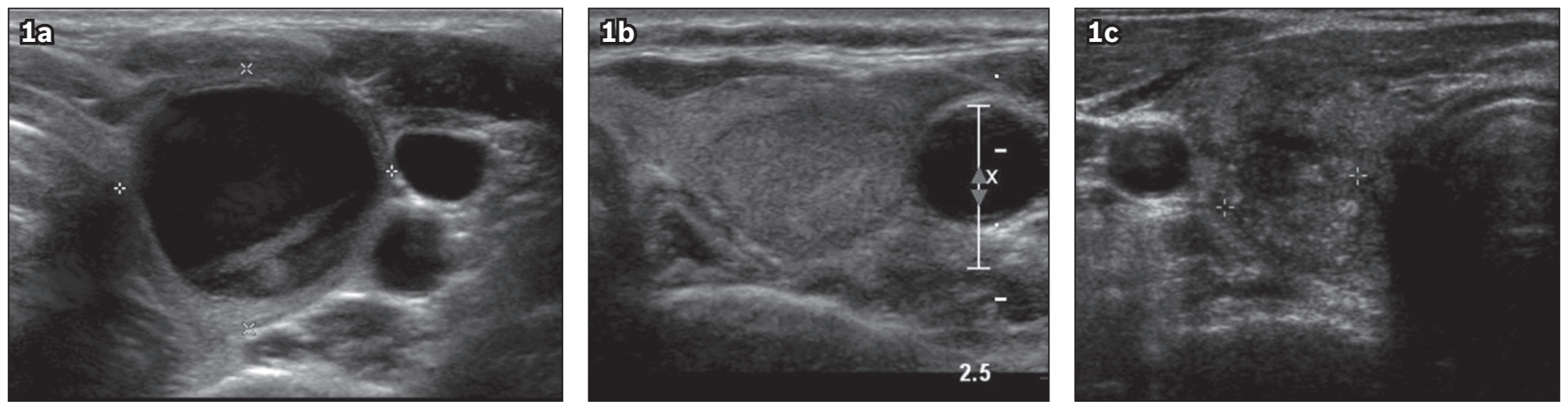

Fig. 1 US images show the three sonographic patterns. (a) Left thyroid cyst, classified as ATA benign, BTA U2 and TIRADS 3 , was shown to be a haemorrhagic cyst on histology. (b) Left thyroid solid and hypoechoic nodule, classified as ATA intermediate suspicion, BTA U4 and TIRADS 4B, was shown to be a benign nodular goitre on histology. (c) Right thyroid solid, hypoechoic nodule with taller-than-wide shape, irregular margins and microcalcifications, classified as ATA high suspicion, BTA U5 and TIRADS 5, was shown to be papillary thyroid cancer on histology. ATA: American Thyroid Association Management Guidelines; BTA: British Thyroid Association Guidelines for the Management of Thyroid Cancer; TIRADS: Thyroid Imaging Reporting and Data System

based on the 2009 ATA guidelines. ${ }^{(10)}$ Of these thyroid nodules, those that were surgically resected and measured more than $1 \mathrm{~cm}$ were studied retrospectively. Information such as the gender and age of the patient during the US-FNA preceding surgery, as well as cytology and histopathology results, were collected from the hospital's electronic medical case records and case notes.

At our institution, cytological results were broadly classified according to the Bethesda System for Reporting Thyroid Cytopathology ${ }^{(11)}$ into the following categories: non-diagnostic; benign; atypia of undetermined significance or follicular lesion of undetermined significance; follicular neoplasm or suspicious for a follicular neoplasm; suspicious for malignancy; or malignant. In addition, static US images and radiologists' reports of each resected nodule were reviewed by two independent observers with 2-8 years' experience in US-FNA to ensure the consistent assignment of US risk category to each nodule. In the event of disagreements between observers, they reviewed the US images together to reach a consensus.

All nodules were assessed for the following features: size; composition; hypoechogenicity; microlobulated or irregular margins; taller-than-wide shape; microcalcifications; macrocalcifications; disrupted rim calcifications; intranodular vascularity; and abnormal cervical lymphadenopathy. Size was measured at the maximum dimension. The composition of the nodule was classified as purely cystic, mixed or solid, based on the ratio of the cystic to the solid portion of the nodule. The echogenicity of the nodule was compared with that of the surrounding parenchyma, and marked hypoechogenicity was defined as lower echogenicity than that of the cervical strap muscle. Echogenicity of a mixed solid-cystic nodule was assessed based on the solid portion. Microcalcifications were defined as tiny $(<1 \mathrm{~mm}$ in diameter), punctate and hyperechoic foci with or without acoustic shadows, while macrocalcifications were defined as $>1 \mathrm{~mm}$ in diameter. A taller-than-wide shape was defined as one having anteroposterior to transverse diameter ratio $\geq 1$. Colour Doppler US images were assessed for intranodular vascularity, and this was classified on a four-point scale as suggested by Fukunari et al, with Grades 3 and 4 considered to be high intranodular vascularity. ${ }^{(12,13)}$ Abnormal cervical lymph nodes were defined as those that exhibit the following features: hyperechogenicity; cystic; peripheral vascularity; microcalcifications; or round shape. ${ }^{(14)}$

The US appearances of these resected nodules were then classified based on the recent ATA guidelines, BTA guidelines and TIRADS classification. ${ }^{(3,4,6)}$ The three US risk stratification systems are shown in the Appendix. The ATA guidelines classify the US appearance of thyroid nodules into benign or low, intermediate or high suspicion of malignancy. The BTA guidelines classify the US appearance of thyroid nodules as U1 (normal), U2 (benign), U3 (indeterminate), U4 (suspicious) or U5 (malignant). The number of suspicious US features of each thyroid nodule was also counted based on the TIRADS classification proposed by Kwak et al. ${ }^{(6)}$ In this scoring system, a solid nodule, hypoechogenicity or marked hypoechogenicity, microlobulated or irregular margins, microcalcifications or mixed calcifications, and a taller-than-wide shape were considered suspicious US features. Thyroid nodules without suspicious features (e.g. pure cyst or mixed solid-cystic nodules without any suspicious features) were classified as TIRADS Category 3. Thyroid nodules with one, two, three/four or five suspicious features were classified as Categories 4A, 4B, 4C and 5, respectively. Examples of US images of thyroid nodules classified based on the three risk stratification systems are showed in Fig. 1. The study was approved by the SingHealth Centralised Institutional Review Board.

Data was analysed using IBM SPSS Statistics for Windows version 21.0 (IBM Corp, Armonk, NY, USA). Baseline continuous data was expressed as mean \pm standard deviation, and categorical data was expressed in percentages. Chi-square or Fisher's exact test was used to test the association between the outcome and categorical variables. $t$-test was used to test differences in the means of continuous variables by the outcome. The diagnostic performance of the three US risk classification systems (i.e. sensitivity, specificity, PPV and negative predictive value [NPV]) was calculated. A p-value $<0.05$ was considered as statistically significant.

\section{RESULTS}

We studied 167 thyroid nodules from 150 patients. The nodules were resected surgically during the five-and-a-half-year study period. Surgical resection of these thyroid nodules revealed 
115 benign and 52 malignant histologies (34 papillary thyroid, 14 follicular thyroid, two medullary thyroid and two anaplastic thyroid cancers). There were no significant differences in age $(53.6 \pm 13.0$ years vs. $54.4 \pm 12.4$ years; $p=0.735)$ or gender distribution ( $88.1 \%$ female vs. $83.7 \%$ female; $p=0.453$ ) between patients with benign nodules and those with malignant nodules.

Surgery was performed on 87 nodules with benign cytologic findings from 77 patients for the following reasons: suspicious US findings $(n=20)$; thyroid nodules showing substantial growth $(\mathrm{n}=6)$; and compression symptoms from large $(>5 \mathrm{~cm})$ multinodular goitres or nodules $(n=26)$. Eight patients had surgery to treat their underlying Graves' disease or toxic multinodular

Table I. Comparison of ultrasonography (US) characteristics between thyroid nodules with benign and malignant histology outcomes.

\begin{tabular}{|llll|}
\hline \multirow{2}{*}{ US characteristic } & \multicolumn{2}{c}{ No. (\%) } & p-value \\
\cline { 2 - 3 } & $\begin{array}{l}\text { Benign } \\
\text { (n= 112) }\end{array}$ & $\begin{array}{l}\text { Malignant } \\
\text { (n= 51) }\end{array}$ & \\
\hline Largest diameter* $(\mathrm{cm})$ & $3.1 \pm 1.5$ & $3.0 \pm 1.7$ & 0.528 \\
\hline Solid nodule & $70(62.5)$ & $40(78.4)$ & $0.049^{+}$ \\
\hline Hypoechogenicity & $32(28.6)$ & $36(70.6)$ & $<0.001^{+}$ \\
\hline Irregular margins & $9(8.0)$ & $18(35.3)$ & $<0.001^{\dagger}$ \\
\hline Taller-than-wide morphology & $3(2.7)$ & $5(9.8)$ & $0.031^{+}$ \\
\hline Microcalcification & $9(8.0)$ & $17(33.3)$ & $<0.001^{\dagger}$ \\
\hline Macrocalcification & $26(23.2)$ & $11(21.6)$ & 1.000 \\
\hline Disrupted rim calcification & $1(0.9)$ & $5(9.8)$ & $0.012^{\dagger}$ \\
\hline Intranodular vascularity & $14(12.5)$ & $12(23.5)$ & 0.105 \\
\hline Cervical lymph node & $1(0.9)$ & $7(13.7)$ & $0.001^{+}$ \\
\hline
\end{tabular}

*Data presented as mean \pm standard deviation. TStatistically significant. goitres, and 17 patients elected surgery as the method of treatment for their thyroid nodules. Another nine patients who had nondiagnostic FNA cytology results also underwent surgery - five had suspicious US findings and four opted for surgical resection of their thyroid nodules.

There were four cases with no US images. Three cases could not be assigned an ATA or a BTA classification: two nodules were solid-cystic in nature, but one had a taller-than-wide shape and the other had microcalcifications (both benign on histology), while the third nodule was solid and isoechoic on US but had associated suspicious cervical lymphadenopathy (papillary thyroid cancer on histology). A comparison of individual sonographic characteristics between thyroid nodules with benign histologies and those with malignant histologies is presented in Table I. There was no significant difference in the size of benign thyroid nodules compared to malignant ones. Compared with benign nodules, significantly higher percentages of malignant nodules were solid (78.4\% vs. $62.5 \%$; $p=0.049)$ or hypoechoic $(70.6 \%$ vs. $28.6 \%$; $\mathrm{p}<0.001)$, had irregular margins $(35.3 \%$ vs. $8.0 \% ; \mathrm{p}<0.001)$, taller-than-wide morphology $(9.8 \%$ vs. $2.7 \%$; $p=0.031)$, microcalcifications $(33.3 \%$ vs. $8.0 \%$; $<<0.001)$, disrupted rim calcifications ( $9.8 \%$ vs. $0.9 \%$; $p=0.012)$ or associated abnormal cervical lymphadenopathy $(13.7 \%$ vs. $0.9 \%$; $p=0.001)$.

The malignancy rates of the ATA categories were benign $0 \%$, very low suspicion $5.9 \%$, low suspicion $14.3 \%$, intermediate suspicion $40.0 \%$ and high suspicion $67.5 \%$ (Table II), with significant differences among the various categories $(p<0.001)$. The malignancy rates of the BTA categories were U2 8.2\%, U3 22.6\%, U4 44.8\%, and U5 64.1\% ( $p<0.001$ ). Based on the TIRADS classification, the malignancy rates for Categories 3, 4A,

Table II. Malignancy rates of thyroid nodules based on sonographic patterns and recommended malignancy rates based on the literature.

\begin{tabular}{|c|c|c|c|c|c|}
\hline \multirow[t]{2}{*}{ Classification } & \multirow{2}{*}{$\begin{array}{l}\text { No. of cases } \\
(n=160)\end{array}$} & \multicolumn{2}{|c|}{ No. (\%) } & \multirow{2}{*}{$\begin{array}{l}\text { Malignancy } \\
\text { rate (\%) }\end{array}$} & \multirow{2}{*}{$\begin{array}{l}\text { Recommended } \\
\text { malignancy } \\
\text { rate (\%) }\end{array}$} \\
\hline & & $\begin{array}{l}\text { Benign } \\
(n=110)\end{array}$ & $\begin{array}{l}\text { Malignant } \\
(n=50)\end{array}$ & & \\
\hline \multicolumn{6}{|l|}{ ATA } \\
\hline Benign & 3 & $3(2.7)$ & 0 & 0 & $<1$ \\
\hline Very low suspicion & 17 & $16(14.5)$ & $1(2.0)$ & 5.9 & $<3$ \\
\hline Low suspicion & 70 & $60(54.5)$ & $10(20.0)$ & 14.3 & $5-10$ \\
\hline Intermediate suspicion & 30 & $18(16.4)$ & $12(24.0)$ & 40.0 & $10-20$ \\
\hline High suspicion & 40 & $13(11.8)$ & $27(54.0)$ & 67.5 & $>70-90$ \\
\hline \multicolumn{6}{|l|}{ BTA } \\
\hline Benign (U2) & 61 & $56(50.9)$ & $5(10.0)$ & 8.2 & \\
\hline Intermediate (U3) & 31 & $24(21.8)$ & $7(14.0)$ & 22.6 & \\
\hline Suspicious (U4) & 29 & $16(14.5)$ & $13(26.0)$ & 44.8 & \\
\hline Malignant (U5) & 39 & $14(12.7)$ & $25(50.0)$ & 64.1 & \\
\hline \multicolumn{6}{|l|}{ TIRADS } \\
\hline 3 & 34 & $31(28.2)$ & $3(6.0)$ & 8.8 & 1.7 \\
\hline $4 \mathrm{~A}$ & 46 & $39(35.5)$ & $7(14.0)$ & 15.2 & 3.3 \\
\hline $4 B$ & 47 & $30(27.3)$ & $17(34.0)$ & 36.2 & 9.2 \\
\hline $4 C$ & 32 & $10(9.1)$ & $22(44.0)$ & 68.8 & $44.4-72.4$ \\
\hline 5 & 1 & $0(0)$ & $1(2.0)$ & 100.0 & 87.5 \\
\hline
\end{tabular}

BTA did not provide recommended malignancy rates for each $U$ category in the guidelines. ATA: American Thyroid Association Management Guidelines; BTA: British Thyroid Association Guidelines for the Management of Thyroid Cancer; TIRADS: Thyroid Imaging Reporting and Data System 
$4 \mathrm{~B}, 4 \mathrm{C}$ and 5 were $8.8 \%, 15.2 \%, 36.2 \%, 68.8 \%$ and $100.0 \%$, respectively $(p<0.001)$. The diagnostic performance of the ATA guidelines when considering benign and very-low-suspicion US patterns as negative test outcomes and low-to-high-suspicion nodules as positive test outcomes was as follows: sensitivity $98.0 \%$, specificity $17.3 \%$, PPV $35.0 \%$ and NPV $95.0 \%$. The US appearances that were benign and very low suspicion were considered negative test outcomes in our study because the ATA guidelines recommend no FNA and observation without further intervention, respectively, for these two categories. The diagnostic performance of the BTA guidelines when considering $U 2$ as a negative test outcome and U3-U5 as positive test outcomes was as follows: sensitivity $90 \%$, specificity $50.9 \%$, PPV $45.5 \%$ and NPV $91.8 \%$. The diagnostic performance of the TIRADS classification when considering Category 3 as a negative test outcome and Categories 4A-5 as positive test outcomes was as follows: sensitivity $94.0 \%$, specificity $28.2 \%$, PPV $37.3 \%$ and NPV $91.2 \%$.

\section{DISCUSSION}

US is an important diagnostic tool in predicting thyroid malignancy and selecting thyroid nodules that should be evaluated by FNA. In our study, there were higher percentages of malignant nodules that were solid and had irregular margins, hypoechogenicity and taller-than-wide morphology, microcalcifications, disrupted rim calcifications or associated abnormal cervical lymphadenopathy. These US features were also included in the ATA, BTA and TIRADS guidelines. Nodule size and the presence of macrocalcifications and intranodular vascularity were not significantly different between benign and malignant nodules. In a recent meta-analysis conducted to determine the accuracy of US features in predicting malignancy, Brito et al found that thyroid nodule size was not an accurate predictor of thyroid cancer across different size cut-offs. ${ }^{(15)}$ The presence of intranodular macrocalcifications was also not consistently associated with thyroid cancer in previous studies. ${ }^{(16,17)}$ However, the presence of disrupted rim calcifications, which suggests tumour invasion in the area of disrupted calcification, is a feature associated with malignancy. ${ }^{(18)}$ In a retrospective study of 1,083 thyroid nodules, Moon et al reported that intranodular vascularity was seen in $31 \%$ of benign thyroid nodules compared to $17 \%$ of malignant nodules. The authors thus concluded that this sonographic feature alone, or in combination with other suspicious malignant features on grayscale US, is not useful in predicting thyroid malignancy. ${ }^{(19)}$ However, intranodular vascularity may correlate better with malignancy in follicular thyroid cancers compared to papillary thyroid cancers. ${ }^{(15,20,21)}$ Although individual US features do not provide strong evidence to confirm or rule out a diagnosis of malignancy, ${ }^{(22,23)}$ the combination of several suspicious US features into sonographic patterns improves the prediction of malignancy risk in these nodules.

In the present study, the malignancy rates of the thyroid nodules increased with increasing suspicious sonographic patterns based on the ATA, BTA and TIRADS categories. The malignancy rates in our study were higher than those recommended by the current literature for both the ATA guidelines and TIRADS classification (Table II). The overall malignancy rate in our study was also high, at $31.7 \%$. Since this is a retrospective analysis of a highly selected group of patients undergoing thyroidectomy, these thyroid nodules may already be at higher risk for malignancy based on the managing physician's clinical impression. All three thyroid nodules that could not be assigned an ATA or a BTA classification would be classified as TIRADS Category 4A based on the number of suspicious US features (which excluded associated suspicious cervical lymphadenopathy) and considered low suspicion for malignancy. However, on histology, one of these three thyroid nodules was found to be a papillary thyroid cancer. A recent study noted that the malignancy risk was $18.2 \%$ for thyroid nodules that could not be classified into a specific pattern based on the latest ATA guidelines. ${ }^{(24)}$ Hence, although isolated suspicious US features cannot predict the risk of malignancy, they should be taken into consideration when risk stratifying nodules that do not fit into particular sonographic patterns based on current guidelines. Our study also highlighted a few specific issues with the ATA guidelines that may need to be addressed, such as potentially worrisome features of malignancy that are not included among the patterns in the guidelines. ${ }^{(25)}$

The present study compared the diagnostic performance of three established US risk classification guidelines. Sensitivity and NPV were $\geq 90 \%$ for all three guidelines. Among them, the ATA guidelines had the highest sensitivity and NPV. Notably, none of the thyroid nodules with sonographic patterns that were considered benign, and only one solid-cystic thyroid nodule with a sonographic pattern associated with very low suspicion for malignancy, turned out to be malignant on eventual histology. The histology of this nodule was a 1.8-cm minimally invasive follicular thyroid cancer. In addition, out of the ten malignant nodules that were classified as low suspicion by ATA sonographic patterns, six were follicular thyroid cancers, three were papillary thyroid cancers and one was a medullary thyroid cancer. Studies have suggested that follicular thyroid cancers may exhibit differences in sonographic appearance compared to papillary thyroid cancers, and may also have some features in common with follicular adenoma. ${ }^{(26,27)}$ Although specificity and PPV were higher with the BTA guidelines and TIRADS classification compared to the ATA guidelines, five nodules based on the BTA guidelines and three nodules based on the TIRADS classification, which were considered to have benign sonographic patterns, were found to be thyroid cancers on final histology. The results of our study confirmed the important role of sonographic patterns as a screening tool in identifying thyroid nodules warranting FNA, and suggest that US alone may not be specific enough to discern benign nodules from malignant ones.

The main limitations of the present study were its retrospective design and small numbers. Also, physicians reading the US images were not blinded to the clinical information available, such as cytology and histology findings. Each US image was read independently by two observers, who then assigned a sonographic pattern based on the pictorial classification outlined by the guidelines used in this study. Although we did not study 
the interobserver correlation, two recent studies have reported moderate to substantial interobserver correlation for identification of nodule sonographic patterns. ${ }^{(23,28)}$ We also did not have clinical information on risk factors for thyroid cancer, such as previous head and neck irradiation or family history of thyroid cancer, which might have influenced the decision to surgically remove the thyroid nodule.

The strength of the present study lies in the fact that only nodules that were resected were included and the final histology was used as the reference standard, since the histology provides the greatest certainty on the eventual diagnosis. Previous studies that compared the diagnostic performance of different US guidelines had included only nodules that were diagnosed based on cytologic findings. ${ }^{(24,29)}$ This could result in some malignant nodules being missed and lead to potential bias in the results. This is also the only local study to date that systematically examines the US features of thyroid nodules using established US risk stratification systems.

In conclusion, the results of the present study showed that sonographic patterns outlined by the ATA guidelines, BTA guidelines and TIRADS classification have the high sensitivity and NPV required for triaging of thyroid nodules for subsequent FNA. It is also important to note that current US criteria do not replace FNA in establishing a definitive diagnosis in thyroid nodules. Several recent studies have suggested using the potential combination of US patterns, cytology results from FNA and molecular markers in enhancing stratification of the malignant risk of thyroid nodules before recommending surgical resection, especially in nodules with indeterminate cytology outcomes..$^{(7,30-32)}$ However, molecular markers are yet to be widely available or affordable at this point. The findings of our study also suggest that although isolated suspicious US features cannot predict the risk of malignancy, they should be taken into account when risk stratifying nodules that do not fit into particular sonographic patterns based on the current guidelines.

\section{REFERENCES}

1. Can AS, Peker K. Comparison of palpation-versus ultrasound-guided fine-needle aspiration biopsies in the evaluation of thyroid nodules. BMC Res Notes 2008; 1:12.

2. Cesur M, Corapcioglu D, Bulut S, et al. Comparison of palpation-guided fineneedle aspiration biopsy to ultrasound-guided fine-needle aspiration biopsy in the evaluation of thyroid nodules. Thyroid 2006; 16:555-61.

3. Haugen BR, Alexander EK, Bible KC, et al. 2015 American Thyroid Association Management Guidelines for Adult Patients with Thyroid Nodules and Differentiated Thyroid Cancer: The American Thyroid Association Guidelines Task Force on Thyroid Nodules and Differentiated Thyroid Cancer. Thyroid 2016; 26:1-133.

4. Perros P, Boelaert K, Colley S, et al; British Thyroid Association. Guidelines for the management of thyroid cancer. Clin Endocrinol (Oxf) 2014; 81 Suppl 1:1-122.

5. Horvath E, Majlis S, Rossi R, et al. An ultrasonogram reporting system for thyroid nodules stratifying cancer risk for clinical management. J Clin Endocrinol Metab 2009; 94:1748-51.

6. Kwak JY, Han KH, Yoon JH, et al. Thyroid imaging reporting and data system for US features of nodules: a step in establishing better stratification of cancer risk. Radiology 2011; 260:892-9.

7. Chng CL, Kurzawinski TR, Beale T. Value of sonographic features in predicting malignancy in thyroid nodules diagnosed as follicular neoplasm on cytology.
Clin Endocrinol (Oxf) 2015; 83:711-6.

8. Moon HJ, Kim EK, Yoon JH, Kwak JY. Malignancy risk stratification in thyroid nodules with nondiagnostic results at cytologic examination: combination of thyroid imaging reporting and data system and the Bethesda System. Radiology 2015; 274:287-95.

9. Moon HJ, Kim EK, Kwak JY. Malignancy risk stratification in thyroid nodules with benign results on cytology: combination of thyroid imaging reporting and data system and Bethesda system. Ann Surg Oncol 2014; 21:1898-903.

10. American Thyroid Association (ATA) Guidelines Taskforce on Thyroid Nodules and Differentiated Thyroid Cancer, Cooper DS, Doherty GM, et al. Revised American Thyroid Association management guidelines for patients with thyroid nodules and differentiated thyroid cancer. Thyroid 2009; 19:1167-214.

11. Cibas ES, Ali SZ. The Bethesda System for reporting thyroid cytopathology. Thyroid 2009; 19:1159-65.

12. Fukunari N, Nagahama M, Sugino K, et al. Clinical evaluation of color Doppler imaging for the differential diagnosis of thyroid follicular lesions. World J Surg 2004; 28:1261-5.

13. Baskin HJ, Sr, Duick DS, Levine RA. Thyroid Ultrasound and Ultrasound-Guided FNA Biopsy. New York: Springer-Verlag; 2013.

14. Leenhardt L, Erdogan MF, Hegedus L, et al. 2013 European thyroid association guidelines for cervical ultrasound scan and ultrasound-guided techniques in the postoperative management of patients with thyroid cancer. Eur Thyroid J 2013; 2:147-59.

15. Brito JP, Gionfriddo MR, Al Nofal A, et al. The accuracy of thyroid nodule ultrasound to predict thyroid cancer: systematic review and meta-analysis. J Clin Endocrinol Metab 2014; 99:1253-63.

16. Wang N, Xu Y, Ge C, Guo R, Guo K. Association of sonographically detected calcification with thyroid carcinoma. Head Neck 2006; 28:1077-83.

17. Shi C, Li S, Shi T, et al. Correlation between thyroid nodule calcification morphology on ultrasound and thyroid carcinoma. J Int Med Res 2012; 40:350-7.

18. Park YJ, Kim JA, Son EJ, et al. Thyroid nodules with macrocalcification: sonographic findings predictive of malignancy. Yonsei Med J 2014; 55:339-44.

19. Moon HJ, Kwak JY, Kim MJ, Son EJ, Kim EK. Can vascularity at power Doppler US help predict thyroid malignancy? Radiology 2010; 255:260-9.

20. Papini E, Guglielmi R, Bianchini A, et al. Risk of malignancy in nonpalpable thyroid nodules: predictive value of ultrasound and color-Doppler features. J Clin Endocrinol Metab 2002; 87:1941-6.

21. Cappelli C, Castellano M, Pirola I, et al. The predictive value of ultrasound findings in the management of thyroid nodules. QJM 2007; 100:29-35.

22. Moon WJ, Jung SL, Lee JH, et al; Thyroid Study Group, Korean Society of Neuro- and Head and Neck Radiology. Benign and malignant thyroid nodules: US differentiation--multicenter retrospective study. Radiology 2008; 247:762-70.

23. Remonti LR, Kramer CK, Leitão CB, Pinto LC, Gross JL. Thyroid ultrasound features and risk of carcinoma: a systematic review and meta-analysis of observational studies. Thyroid 2015; 25:538-50

24. Yoon JH, Lee HS, Kim EK, Moon HJ, Kwak JY. Malignancy risk stratification of thyroid nodules: comparison between the thyroid imaging reporting and data system and the 2014 American Thyroid Association management guidelines. Radiology 2016; 278:917-24.

25. Malhi H, Grant EG. Both TIRADS and the ATA guidelines provide effective malignancy risk stratification for thyroid nodules. Clin Thyroidol 2016; 28:238-40.

26. Jeh SK, Jung SL, Kim BS, Lee YS. Evaluating the degree of conformity of papillary carcinoma and follicular carcinoma to the reported ultrasonographic findings of malignant thyroid tumor. Korean J Radiol 2007; 8:192-7.

27. Hoang JK, Lee WK, Lee M, Johnson D, Farrell S. US features of thyroid malignancy: pearls and pitfalls. Radiographics 2007; 27:847-60; discussion 861-5.

28. Ko SY, Lee HS, Kim EK, Kwak JY. Application of the Thyroid Imaging Reporting and Data System in thyroid ultrasonography interpretation by less experienced physicians. Ultrasonography 2014; 33:49-57.

29. Ahn SS, Kim EK, Kang DR, et al. Biopsy of thyroid nodules: comparison of three sets of guidelines. AJR Am J Roentgenol 2010; 194:31-7.

30. De Napoli L, Bakkar S, Ambrosini CE, et al. Indeterminate single thyroid nodule: synergistic impact of mutational markers and sonographic features in triaging patients to appropriate surgery. Thyroid 2016; 26:390-4

31. Yoon JH, Kwon HJ, Kim EK, Moon HJ, Kwak JY. Subcategorization of atypia of undetermined significance/follicular lesion of undetermined significance (AUS/ FLUS): a study applying Thyroid Imaging Reporting and Data System (TIRADS). Clin Endocrinol (Oxf) 2016; 85:275-82.

32. Maia FF, Matos PS, Pavin EJ, Zantut-Wittmann DE. Thyroid imaging reporting and data system score combined with Bethesda system for malignancy risk stratification in thyroid nodules with indeterminate results on cytology. Clin Endocrinol (Oxf) 2015; 82:439-44. 


\section{APPENDIX}

American Thyroid Association (ATA), British Thyroid Association (BTA) and Thyroid Imaging Reporting and Data System (TIRADS) ultrasonography sonographic patterns

\begin{tabular}{|c|c|}
\hline \multicolumn{2}{|l|}{ ATA classification } \\
\hline Benign & Purely cystic nodules (no solid component) \\
\hline Very low suspicion & $\begin{array}{l}\text { Spongiform or partially cystic nodules without any of the sonographic features described in low, } \\
\text { intermediate or high suspicion patterns }\end{array}$ \\
\hline Low suspicion & $\begin{array}{l}\text { Isoechoic or hyperechoic solid nodule or partially cystic nodule with eccentric solid areas, without } \\
\text { microcalcification, irregular margin or extrathyroidal extension (ETE), or taller-than-wide shape }\end{array}$ \\
\hline Intermediate suspicion & $\begin{array}{l}\text { Hypoechoic solid nodule with smooth margins without microcalcifications, ETE or taller-than-wide } \\
\text { shape }\end{array}$ \\
\hline High suspicion & $\begin{array}{l}\text { Solid hypoechoic nodule or solid hypoechoic component of a partially cystic nodule with one or } \\
\text { more of the following features: irregular margins (infiltrative, microlobulated), microcalcifications, } \\
\text { taller-than-wide shape, rim calcifications with small extrusive soft tissue component, evidence of } \\
\text { ETE }\end{array}$ \\
\hline \multicolumn{2}{|l|}{ BTA classification } \\
\hline U1 (normal) & Normal thyroid gland \\
\hline U2 (benign) & $\begin{array}{l}\text { - Spongiform or honeycomb appearance } \\
\text { - Purely cystic nodule and nodules with a cystic component containing colloid (hyperechoic foci } \\
\text { with a'ring-down' sign) } \\
\text { - Egg-shell type calcification around the periphery of a nodule } \\
\text { - Isoechoic or (mildly) hyperechoic nodule, typically with a surrounding hypoechoic halo } \\
\text { - Peripheral vascularity on colour flow or power Doppler }\end{array}$ \\
\hline U3 (intermediate/equivocal) & $\begin{array}{l}\text { - Homogenous solid nodule with marked hyperechogenicity and halo } \\
\text { - Possible hypoechoic nodule with presence of equivocal echogenic foci or cystic change, or } \\
\text { presence of mixed or central vascularity }\end{array}$ \\
\hline U4 (suspicious) & $\begin{array}{l}\text { - Solid, hypoechoic or very hypoechoic nodule } \\
\text { - Solid, hypoechoic nodule with disrupted peripheral calcification or lobulated outline }\end{array}$ \\
\hline U5 (malignant) & $\begin{array}{l}\text { Solid, hypoechoic, lobulated/irregular outline with microcalcification, globular calcification, } \\
\text { intranodular vascularity taller-than-wide shape or characteristics associated with } \\
\text { lymphadenopathy }\end{array}$ \\
\hline \multicolumn{2}{|l|}{ TIRADS classification } \\
\hline TIRADS 3 & No suspicious ultrasonography feature* \\
\hline TIRADS 4A & One suspicious ultrasonography feature \\
\hline TIRADS 4B & Two suspicious ultrasonography features \\
\hline TIRADS 4C & Three or four suspicious ultrasonography features \\
\hline TIRADS 5 & Five suspicious ultrasonography features \\
\hline
\end{tabular}

*Suspicious features are solid nodule, hypoechogenicity or marked hypoechogenicity, microlobulated or irregular margins, microcalcifications or mixed calcifications and a taller-than-wide shape. 